\title{
Physical Therapy Management for a Patient with Delayed Quadriceps Tendon Repair and Secondary, Unilateral Lymphedema: A Case Report
}

\author{
Mijeong Joo-Bunyard, PT, DPT ${ }^{1}$, Man-Soo Ko, PT, PhD ${ }^{* 1}$ \\ ${ }^{1}$ Dept. of Physical Therapy, University of Texas Medical Branch, USA
}

\begin{abstract}
Purpose This case follows the complications, treatment, and results of a 69 years old male patient who sustained complete right quadriceps tendon tear secondary to a fall. Due to cardiovascular complications, surgery was delayed for a month, and reconstructive surgery was extensive. Methods He was assessed as suffering from significantly decreased range of motion (ROM), decreased strength, and secondary, unilateral lymphedema. Physical therapy began 10 weeks post-surgery and consisted of a 3-month rehabilitation program to increase ROM, strength, and balance, as well as to reduce edema. Results Measures were assessed at initial evaluation and final discharge. Right knee Active Range of Motion (AROM) flexion increased from 60 to 86 degrees and extension improved from -26 to -15 degrees. Knee joint circumference was reduced from $49 \mathrm{~cm}$ to $45.4 \mathrm{~cm}$. Lower Extremity Functional scale improved from 13/80 to 35/80. Timed Up and Go improved from 18 seconds with bilateral crutches to 7 seconds without crutches. Tandem balance progressed from less than 1 second to greater than 1 minute. Single Limb Stance from less than 1 second to 29 seconds on the right. Conclusion The case report describes the interventions status post delayed quadriceps tendon repair for an older adult that was effectively in improving AROM, functional mobility, and balance.
\end{abstract}

Key words Quadriceps, Extension mechanism rupture, knee, Lower Extremity Functional scale, Timed Up and Go

Corresponding author Man-Soo Ko (mako@utmb.edu)

Received date 20 Jan 2021

Revised date 06 Feb 2021

Accept date 14 Feb 2021

\section{Introduction}

The quadriceps tendon rupture is a rare encounter in an outpatient rehabilitation facility. It most commonly occurs with trauma. ${ }^{1)}$ The mechanism of injury is usually related to balance and falls, attempts at balance recovery and ruptures of the extensor mechanism. ${ }^{1)}$ The usual presentation is a middle-aged man with a fall injury. ${ }^{2)}$ The condition usually affects patients over 40 with systemic health problems. ${ }^{3)}$ Edema and difficulty with knee extension are further complicating factors that typically accompany the injury. ${ }^{2)}$ Early treatment is critical towards restoring a full range of motion and function. ${ }^{4)}$ Due to the rarity of the injury, rehabilitation of quadriceps repair has not been studied extensively. While the common presentation is one-sided, factors such as rheumatoid arthritis, diabetes, or

http:dx.doi.org/10.17817/2021.02.06.1111627 gout, increase the risk for bilateral occurrence. ${ }^{5)}$ Other factors including prolonged use of corticosteroids, fluoroquinolones antibiotic usage, and renal failure can contribute to injury severity. ${ }^{6}$ Although imaging provides confirmation of diagnosis, presentation of the clinical diagnostic triad includes acute pain, inability to extend the knee and suprapatellar gap, lends to a presumptive diagnosis. $\left.{ }^{7}, 8\right)$ Additional signs may include atrophy and decreased strength of the quadriceps of the affected limb. ${ }^{1)}$

Prompt repair of the extensor mechanism in the affected limb is important. However, due to the rarity of this injury, there remains no consensus regarding the best method to repair the quadriceps tendon. ${ }^{4}$ A study by Serino et al. ${ }^{4)}$ shows that there is no superior repair to conserve the mass and the strength of the quadriceps muscles. In contrast, a study conducted by Boudissa et al. $^{3)}$ demonstrates that most of the patients with extensor mechanism repairs were able to achieve functional range of motion of the extension 
and flexion on the affected knee. If the repair is delayed, however, it can impact patients' functional level and quality of activities of daily living (ADLs).

With regards to proper rehabilitation, there is an on-going debate regarding the benefits and drawbacks between early mobilization versus delayed mobilization in order to improve function and ADLs. A study by Serino et al. ${ }^{4}$ shows that early mobilization has increased association with higher adverse effects and financial costs than late mobilization; in the early mobilization group, there was an increased rate of adverse event and a higher rate of re-surgery than the late mobilization group. The study shows a $7 \%$ increase in re-surgery rate in the early mobilization group and a $9 \%$ higher adverse event rate in the early mobilization group when compared to the late mobilization group. ${ }^{4)}$ The period of traditional immobilization is 6-10 weeks post-surgery. While there is speculation that only 3-6 weeks of immobilization suffices, the study concluded that at least 6 weeks of immobilization provides more beneficial outcomes to patients. However, these benefits do not apply to functional outcomes. ${ }^{4}$

Despite a current tendency among therapists to encourage early mobilization treatment, the functional outcomes between patients with early mobilization and traditional rehabilitation protocol do not significantly differ." Although in some cases, patients in the early mobilization groups returned to work a few days earlier, due to the complexity of the repair, the majority of surgeons preferred at least 6 weeks of immobilization. ${ }^{9)}$ However, while the Langenhan study concluded that early mobilization is safe to apply to patients with quadriceps tendon repair, the higher adverse event rate previously mentioned lends weight to the traditional methodology. ${ }^{4,9)}$

While a small body of evidence is available to physical therapists regarding patients with quadriceps tendon repair, most studies are based on acute quadriceps muscle/tendon repair. In most studies, patients with delayed quadriceps tendon rupture repair or with other complications were excluded. Edema and difficulty with knee extension are further complicating factors that typically accompany the injury. ${ }^{2}$ Furthermore, due to the rarity of the injury, rehabilitation of quadriceps repair has not been extensively studied. The purpose of this case report is to describe applied physical therapy interventions for a patient with a delayed repair of complete unilateral quadriceps tendon rupture, along with the development of secondary lymphedema. The goals of the care plan were to increase the patient's range of motion, strength and balance and reduce edema in an outpatient rehabilitation setting.

\section{Material and Methods}

\section{Patient History and clinical impression}

The patient was a 69-year-old Caucasian male, with pertinent medical history, including quadruple bypass, hypertension, rheumatoid arthritis, anxiety, and left hamstring injury with the development of secondary lymphedema a few years ago. He lived alone in a single-level house. However, patient's daughter provided occasional assistance. Approximately 10 weeks prior to his initial visit, patient fell on a flexed knee when getting out of a bathtub causing complete rupture of the right quadriceps' tendon. Due to cardiac issues, the repair was delayed for one month. He was immobilized for four weeks prior to surgery and for six weeks post-operative repair. At initial evaluation, patient exhibited knee flexion contractures and lymphedema. His plan of care targeted range of motion (flexion and extension), edema reduction, and bilateral lower extremity weakness. His goal for physical therapy was to return to his prior level of independence as an independent community ambulator without crutches. Patient received three months of physical therapy to address his deficits.

The subject was referred to physical therapy following a quadriceps tendon repair with weight bearing as tolerated precautions. Upon initial evaluation, patient presented with bilateral axillary crutches and reported that other comorbidities were medically managed. Based on the right knee flexion contracture, edema, and abnormal gait, He was evaluated in the following areas to take the baseline level and to de- 
termine appropriate exercises: range of motion, lower extremity strength, muscle length, knee joint circumference, gait analysis, balance and fall risk. Upon examination, the patient was anxious about returning to his prior level of function. He guarded his involved leg and utilized bilateral axillary crutches while ambulating. His cardiac condition remained stable, and he did not present with other comorbidities. Objective findings and outcome measures showed that the subject had significant bilateral weakness in the lower extremities (right worse than left), limited right knee range of motion, increased risk for falls and abnormal gait pattern. Therefore, therapists assessed that the patient would benefit from physical therapy to improve right knee range of motion, manage lymphedema, increase strength and endurance, and reduce fall risks and gait deficits.

\section{Examination}

1) Lower Extremity Functional Scale (LEFS)

The Lower Extremity Functional Scale (LEFS) is a reliable and validated patient report outcome measures. ${ }^{10}$, 11) It consists of 20 questions and measures lower extremity function in a wide variety of disorders and treatment. Both the minimal detectable change (MDC) and the minimal clinically important difference (MCID) were 9 points. ${ }^{12)}$ LEFS was assessed at the initial evaluation, every 10 visits or 30 days after, and at the discharge. His initial score was 13 out of 80 , suggesting very limited functional level.

2) Knee joint circumference, range of motion and MMT Due to a one-month delay of the surgery, the patient was immobilized for a total of 10 weeks prior to be- ginning physical therapy. M. Boudissa et al. ${ }^{3)}$ found that if the quadriceps tendon rupture is repaired in a timely manner, most patients return to a prior level of functionality with intensive physical therapy at least over 6 weeks. Their study showed that 97\% of patients were able to reach 125.1 degrees of knee flexion and 0 degrees of extension by a one-year followup. ${ }^{3)}$ While there is no current standard immobilization timeframe, Serino et al. $^{4}$ found that immediate, or early mobilization increases the risk of adverse effects compared to patients immobilized for at least six weeks.

A study by Stolldorf et al. ${ }^{13)}$ shows that patients with secondary lymphedema of leg with non-cancer causes experience similar level of psychosocial distress as those with primary lymphedema with cancer. Also, they all suffer from swelling, pain, stiffness, and decreased mobility. ${ }^{13)}$ Upon initial examination, the patient's right knee flexion was 60 degrees and right knee extension was lacking 26 degrees from 0 . Right knee joint circumference was $49 \mathrm{~cm}$ vs. left was 45 $\mathrm{cm}$. As expected, due to prolonged immobilization, the patient's bilateral hip extension and abduction were weak. Patient's right knee extensor and flexor strength were significantly less than left side. (Table 1)

\section{3) The Timed Up and Go (TUG)}

The Timed Up and Go test is a valid and reliable tool to predict fall risk in elderly with MDC of 2.27 in patients with TKA and assess fall risk in elderly. ${ }^{14)}$ Although the TUG has not been validated for quadriceps repair, it is useful assessing high fall risk ( $>13.5$ sec). ${ }^{15)}$ In this case study, the subject performed TUG with bilateral axillary crutches. During the TUG, he

Table 1. Manual Muscle Testing results

\begin{tabular}{ccccc}
\hline & Week 1 & & Week 12 & Right \\
\cline { 2 - 4 } Hip extensors & Right & Left & $4+/ 5$ & $4+/ 5$ \\
Hip abductors & $2+/ 5 *-/ 5$ & $3-/ 5$ & $5 / 5$ & $4+/ 5$ \\
Quadriceps & $2+/ 5 *$ & $5 / 5$ & $4+/ 5$ & $5 / 5$ \\
Hamstrings & $2+/ 5 *$ & $5 / 5$ & $4+/ 5$ & $5 / 5$ \\
\hline
\end{tabular}

\footnotetext{
* pain
} 
exhibited abnormal transitional movement and gait pattern such as antalgic gait, decreased speed, reduced stance time of right lower extremity, multiple steps with turning, forward trunk, impaired knee and hip flexion during swing, lack extension during stance phase, and impaired initial contact. He completed the test in 18 seconds with his crutches, confirming a moderate fall risk.

\section{4) Tandem Stance Balance}

Although the protocols of tandem balance are not standardized, it captures characteristics of standing balance and can be documented with types of assist or lack of assistance, and careful interpretation of results. ${ }^{16)}$ The test duration is generally is either 10 or 30 seconds, with 30 seconds providing the most meaningful predictive capabilities for determining functionality in subjects. ${ }^{16)}$ For this case study, the subject was asked to take a tandem stance with right foot in front and left foot back, with eyes open and without arm assist. Subject was unable to perform tandem more than 1 second, in both with right foot in front and left foot in front.

\section{5) Single Limb Stance (SLS)}

The single limb stance test can be performed with eyes open or eyes closed to assess static balance. While its reliability is not fully established, it has good validity in the relationship with gait performance, fall, frailty, and ADL performance. ${ }^{17)}$ Springer's study ${ }^{18)}$ confirmed the direct, converse relationship between age and unipedal balance, however, balance was not specifically related to gender. With SLS test, the patient attempted to stand on a single limb, however resumed bilateral lower extremity support less than 1 second, without UE support and with eyes open. It suggested the patient's poor balance and fall risk.

\section{Intervention}

He participated in a 60 minutes session, 3 times per week for 12 weeks. Each session was comprised of manual therapy to decrease edema and increase joint mobility, range of motion exercises, endurance training, lower extremity strengthening, and balance. ROM exercises included heel slides that progressed to wall slides with ankle weight. A knee extension device (Extensionator) and prone knee hang with ankle weight were added as the subject improved his tolerance. Manual therapy, such as patella mobilization, proximal and distal tibio-fibular mobilization, iliotibial band (ITB) release, cupping technique, instrument assisted soft tissue mobilization (IASTM) were added to reduce edema and to increase joint mobility.

The patient's secondary lymphedema presented one of the most difficult barriers to regaining full joint mobility. Various interventions were used to manage the secondary lymphedema, such as suggested elevation, exercise, compression, and lymph massage ${ }^{19)}$. During rehabilitation, patient was provided a knee joint flexion and extension range of motion splint device as well as a compression sleeve for secondary lymphedema for home. He was instructed to use the knee splint for at least 1 hour for flexion and 1 hour for extension and wear the compression sleeve throughout the day. Patient education was given in the clinic. However due to the complex don/doff procedures of the device, patient needed total assist. Therefore, patient reported inability to don/doff the device and sleeves due to lack of assist by another person. Patient also claimed an inability to maintain compliance with home program, citing personal reasons. Thus, most of the intervention was performed primarily during the therapy sessions.

Initially, the subject was unable to achieve the ROM required to pedal a stationary bike. Therefore, patient began therapy with heel slides then wall slides with ankle weight up to 5lbs. From week 3-4, patient's ROM improved so that he was able to ride the stationary bike for warm up, decrease stiffness, and increase ROM. As therapy progressed, patient's endurance also increased, which enabled progression of therapeutic activities during sessions. As the patient's cardiac comorbidity affected his endurance with exercises as well as ADLs, this provided a useful secondary benefit.

The goal of the main exercise program was to decrease edema, increase range of motion and improve strength of lower extremities. The first half of sessions 
included stretches of hip flexors, hamstrings, and quadriceps, strengthening exercises such as bridging, clamshells, and single leg raises in three ways on a mat. Manual therapy and other techniques were included to address patellar mobility, ITB shortness, stiffness of muscles and the right knee joint. Techniques included patellar mobilization, IASTM of ITB, quadriceps and hamstrings, cupping on or around the right patella, proximal/distal tibio-fibular mobilization, and Mulligan with movement (knee flexion). For about $21 / 2$ months, patient responded well to the manual therapy and other modalities, however later into the therapy course, patient reported pain symptoms the day following the manual therapy. Subsequently, the frequency of the manual therapy was reduced to respect tissue response.

The last half of therapy session was comprised of standing balance, strengthening and endurance. The course of intervention included gastrocnemius stretch- es, weight shifting laterally and forward/backward, heel raises, modified tandem balance, terminal knee extension, heel to toe gait, and resisted hip abduction and walking. As the program progressed, patient performed exercises utilizing the hip and knee exercise devices to further strengthen the musculature of hip, knees and ankles. Throughout the intervention, patient demonstrated high anxiety, poor motor coordination, and low compliance in home exercise program. However, patient regularly participated in therapy three times per week. Details of intervention are listed in Table 2 .

\section{Results}

All outcome measures were tested at the evaluation, and final measurements were assessed one week prior to discharge. At discharge, the patient demonstrated

\section{Table 2. Interventions performed during week 1 and 12*}

\section{Week 1}

Heel slides/wall slides

R. hip flexor, hamstring, quadriceps stretch

Prone knee extension with weight/ Extensionator device

Bridging

3-way clamshells (hip flexion, abduction and extension)

Single leg raises in supine (hip abduction \& extension)

Gastrocnemius stretch

Weight shifting laterally and front/back with a mirror

Heel raises

Modified Tandem balance

Stool Scoots

Terminal Knee extension

Heel to toe gait at parallel bars

One crutch training

Manual therapy/modalities for 10-15 $\mathrm{min}$.

(Patellar mobilization, proximal/distal tibio-fibular mobilization, IASTM

(for quadriceps, hamstring, ITB), Mulligan mobilization with movement,

Cupping technique for patellar mobility)

\footnotetext{
* Dosage and intensity as well as progression were determined at each therapy session based on the patient's pain symptom report and range of motion and edema status.
} 
Table 3. Outcome Measures

\begin{tabular}{|c|c|c|}
\hline Measures & Pre- intervention & $\begin{array}{l}\text { Post-intervention } \\
\text { (3 months in duration) }\end{array}$ \\
\hline R. Knee AROM flexion & 26-60 degrees & 15-86 degrees \\
\hline R. Knee AROM extension & Lacking 26 degrees & Lacking 15 degrees \\
\hline R. Knee joint circumference & $49 \mathrm{~cm}$ (left knee $45 \mathrm{~cm}$ ) & $45.4 \mathrm{~cm}$ \\
\hline Lower Extremity Functional Scale (LEFS) & $13 / 80$ & $31 / 80$ \\
\hline TUG & $\begin{array}{l}18 \text { seconds with bilateral axillary } \\
\text { crutches }\end{array}$ & 7 seconds without $\mathrm{AD}$ \\
\hline Single Limb Stance & $\begin{array}{l}\text { Right: }<1 \text { second } \\
\text { Left: } 3 \text { seconds }\end{array}$ & $\begin{array}{l}\text { Right } 29 \text { seconds } \\
\text { Left: } 14 \text { seconds }\end{array}$ \\
\hline Tandem balance & $\begin{array}{l}\text { Right leg forward: }<1 \text { second } \\
\text { Left leg forward: }<1 \text { second }\end{array}$ & $\begin{array}{l}\text { Right leg forward: }>1 \text { minute } \\
\text { Left leg forward: }>1 \text { minute }\end{array}$ \\
\hline
\end{tabular}

* For the MMT results, refer to TABLE 1.

increased range of motion of the affected knee; flexion from 26-60 degrees to 15-86 degrees, extension from lacking 26 degrees to lacking 15 degrees. The right knee joint circumference decreased from $49 \mathrm{~cm}$ to $45.4 \mathrm{~cm}$.

The patient's balance significantly improved throughout the therapy course; TUG score improved from 18 seconds with bilateral axillary crutches to 7 seconds without crutches. SLS improved from less than $1 \mathrm{sec}-$ ond to 29 seconds on the right lower extremity. Tandem balance also improved from less than $1 \mathrm{sec}-$ ond to greater than 1 minute on right. Patient was able to perform longer duration; however, test was terminated once he reached 1 minute. Both results indicate reduced risk of falls.

Lastly, LEFS score was assessed in order to investigate his functional improvement. The initial score was $13 / 80$ with score 0 in 10 activities out of 20 . At discharge, he reported 31/80, indicating improved function. The most significant progress was in following functions; getting into or out of the bath, or a car, walking between rooms, and standing for an hour. However, patient reported no progress with running or walking a mile from initial evaluation to discharge.

\section{Discussion}

As physical therapists, it is critical to understand the mechanism of quadriceps tendon rupture and repair and possible complications in order to address patients' deficits. Post-operatively, in an out-patient rehabilitation setting, intended outcomes are increased range of motion, strength, balance and reduced edema, with traditional rehabilitation program along with manual therapy. The purpose of this case report was to describe the rehabilitation process for a patient with unilateral, delayed quadriceps tendon repair with secondary lymphedema.

Therapists achieved most of the established goals. However, the patient's diffuse edema and decreased participation in home program limited further progress, even with continuous patient education. Throughout the treatment program, therapists closely monitored tissue response and communicated with the patient to optimize the therapeutic effects. Additionally, to avoid adverse effects, the dosage and intensity of therapy program was adjusted dependent on the subject's response. Eccentric lowering from steps was added at the later stage of the program, and therapists paid attention to the tissue response to adjust intensity and dosage of the exercises to avoid worsening of edema.

In addition to manual and exercise-based interventions, extensive education was incorporated to ad- 
dress the patient's expectations and anxiety, and encourage compliance with the home exercise program. Although patient's adherence to home program was low, this study still demonstrates the ability of traditional treatment methodology to address the patient's range of motion, strength, balance and functional deficits.

The main limitation of this case report was the lack of lymphedema specific treatment available within the clinic. The difficulties presented by the lack of treatment appeared to significantly impact patient's ability to progress as rapidly as may have otherwise been possible. The swelling of the joint initially prevented him from any exercises at all and only gradually - at a much slower than predicted pace - did he begin to conduct the movements prescribed by the therapist.

This case report shows the progress of rehabilitation of a patient with delayed quadriceps tendon repair complicated by secondary lymphedema and other comorbidities. Despite the absence of lymphedema specific treatment and the subject's lack of participation in the provided home program, the traditional approach to his treatment achieved increased functional mobility and strength. With full treatment for all complications as well as compliance on the part of the patient, even more favorable outcomes could be expected. Due to the scarcity of research in non-acute patients, additional studies would be beneficial to determine proper course of therapy.

\section{References}

1. Ciriello V, Gudipati S, Tosounidis T, et al. Clinical outcomes after repair of quadriceps tendon rupture: A systematic review. Injury. 2012;43(11):1931-8.

2. Nori S. Quadriceps tendon rupture. J Family Med Prim Care. 2018;7(1):257-60.

3. Boudissa M, Roudet A, Rubens-Duval B, et al. Acute quadriceps tendon ruptures: A series of 50 knees with an average follow-up of more than 6 years. Orthop Traumatol Surg Res. 2014;100(2):213-6.

4. Serino J, Mohamadi A, Orman S, et al. Comparison of adverse events and postoperative mobilization following knee extensor mechanism rupture repair: A systematic review and network meta-analysis. Injury. 2017;48(12): 2793-9.

5. Shah MK. Simultaneous bilateral rupture of quadriceps tendons: Analysis of risk factors and associations. South Med J. 2002;95(8):860-6.

6. Grecomoro G, Camarda L, Martorana U. Simultaneous chronic rupture of quadriceps tendon and contra-lateral patellar tendon in a patient affected by tertiary hyperparatiroidism. J Orthop Traumatol. 2008;9(3):15962.

7. Siwek CW, Rao JP. Ruptures of the extensor mechanism of the knee joint. J Bone Joint Surg Am. 1981;63(6):932-7.

8. Spector ED, DiMarcangelo MT, Jacoby JH. The radiologic diagnosis of quadriceps tendon rupture. $\mathrm{N} \mathrm{J}$ Med. 1995;92(9):590-2.

9. Langenhan R, Baumann M, Ricart P, et al. Postoperative functional rehabilitation after repair of quadriceps tendon ruptures: A comparison of two different protocols. Knee Surg Sports Traumatol Arthrosc. 2012;20(11):2275-8.

10. Dingemans SA, Kleipool SC, Mulders MAM, et al. Normative data for the lower extremity functional scale (lefs). Acta Orthop. 2017;88(4):422-6.

11. Mehta SP, Fulton A, Quach C, et al. Measurement properties of the lower extremity functional scale: A systematic review. J Orthop Sports Phys Ther. 2016;46(3):200-16.

12. Binkley JM, Stratford PW, Lott SA, et al. The lower extremity functional scale (lefs): Scale development, measurement properties, and clinical application. North american orthopaedic rehabilitation research network. Phys Ther. 1999;79(4):371-83.

13. Stolldorf DP, Dietrich MS, Ridner SH. A comparison of the quality of life in patients with primary and secondary lower limb lymphedema: A mixed-methods study. West J Nurs Res. 2016;38(10):1313-34.

14. Yuksel E, Kalkan S, Cekmece S, et al. Assessing minimal detectable changes and test-retest reliability of the timed up and go test and the 2-minute walk test in patients with total knee arthroplasty. J Arthroplasty. 2017;32(2): 426-30.

15. Barry E, Galvin R, Keogh C, et al. Is the timed up and go test a useful predictor of risk of falls in community dwelling older adults: A systematic review and meta-analysis. BMC Geriatr. 2014;14:14.

16. Hile ES, Brach JS, Perera S, et al. Interpreting the need 
for initial support to perform tandem stance tests of balance. Phys Ther. 2012;92(10):1316-28.

17. Bohannon RW. Responsiveness of the single-limb stance test. Gait Posture. 2012;35(1):173; author reply 4.

18. Springer BA, Marin R, Cyhan T, et al. Normative values for the unipedal stance test with eyes open and closed. J Geriatr Phys Ther. 2007;30(1):8-15.
19. Kerchner K, Fleischer A, Yosipovitch G. Lower extremity lymphedema update: Pathophysiology, diagnosis, and treatment guidelines. J Am Acad Dermatol. 2008;59(2): 324-31. 